\title{
Studying the Differential Problem with Maple
}

\author{
Chii-Huei Yu \\ Department of Management and Information, Nan Jeon University of Science and Technology, Taiwan \\ *Corresponding Author: chiihuei@mail.nju.edu.tw
}

Copyright $(2013$ Horizon Research Publishing All rights reserved.

\begin{abstract}
This article takes the mathematical software Maple as the auxiliary tool to study the differential problem of two types of trigonometric functions. We can obtain the Fourier series expansions of any order derivatives of these two types of functions by using differentiation term by term theorem, and hence greatly reduce the difficulty of calculating their higher order derivative values. On the other hand, we provide some examples to do calculation practically. The research methods adopted in this study involved finding solutions through manual calculations and verifying these solutions by using Maple. This type of research method not only allows the discovery of calculation errors, but also helps modify the original directions of thinking from manual and Maple calculations.
\end{abstract}

Keywords Derivatives, Trigonometric Functions, Fourier Series Expansions, Differentiation Term By Term Theorem, Maple

\section{Introduction}

The computer algebra system (CAS) has been widely employed in mathematical and scientific studies. The rapid computations and the visually appealing graphical interface of the program render creative research possible. Maple possesses significance among mathematical calculation systems and can be considered a leading tool in the CAS field. The superiority of Maple lies in its simple instructions and ease of use, which enable beginners to learn the operating techniques in a short period. In addition, through the numerical and symbolic computations performed by Maple, the logic of thinking can be converted into a series of instructions. The computation results of Maple can be used to modify our previous thinking directions, thereby forming direct and constructive feedback that can aid in improving understanding of problems and cultivating research interests. Inquiring through an online support system provided by Maple or browsing the Maple website (www.maplesoft.com) can facilitate further understanding of Maple and might provide unexpected insights. As for the instructions and operations of Maple, we can refer to [1-7].

In calculus and engineering mathematics courses, determining the $n$-th order derivative value $f^{(n)}(c)$ of a function $f(x)$ at $x=c$, in general, needs to go through two procedures: firstly finding the $n$-th order derivative $f^{(n)}(x)$ of $f(x)$, and then taking $x=c$ into $f^{(n)}(x)$. These two procedures will make us face with increasingly complex calculations when calculating higher order derivative values of a function (i.e. $n$ is large), and hence to obtain the answers by manual calculations is not easy. In this paper, we mainly evaluate the derivatives of the following two types of trigonometric functions

$$
\begin{aligned}
& f(x)=\frac{a \cos [m(b x+c)]+\lambda^{n} \cos [(m-n)(b x+c)]}{2 a \lambda^{n} \cos [n(b x+c)]+a^{2}+\lambda^{2 n}} \\
& g(x)=\frac{a \sin [m(b x+c)]+\lambda^{n} \sin [(m-n)(b x+c)]}{2 a \lambda^{n} \cos [n(b x+c)]+a^{2}+\lambda^{2 n}}
\end{aligned}
$$

Where $a, b, c, \lambda$ are real numbers, $a, \lambda \neq 0, m$ is any integer, $n$ is any positive integer, and $|\lambda| \neq|a|^{1 / n}$. We can obtain the Fourier series expansions of any order derivatives of trigonometric functions (1) and (2) by using differentiation term by term theorem; these are the major results of this study (i.e., Theorems 1 and 2), and hence greatly reduce the difficulty of calculating their higher order derivative values. As for the study of related differential problems can refer to [8-22]. On the other hand, we propose some examples to do calculation practically. The research methods adopted in this study involved finding solutions through manual calculations and verifying these solutions by using Maple. This type of research method not only allows the discovery of calculation errors, but also helps modify the original directions of thinking from manual and Maple calculations. Therefore, Maple provides insights and guidance regarding problem-solving methods.

\section{Main Results}

Firstly, we introduce a notation and two formulas used in this study.

\subsection{Notation}

Let $z=a+i b$ be a complex number, where 
$i=\sqrt{-1}, a, b$ are real numbers. We denote $a$ the real part of $z$ by $\operatorname{Re}(z)$, and $b$ the imaginary part of $z$ by $\operatorname{Im}(z)$.

\subsection{Euler's Formula}

$e^{i x}=\cos x+i \sin x$, where $x$ is any real number.

\subsection{Geometric Series}

Suppose $z$ is a complex number, and $|z|<1$. Then $\frac{1}{1+z}=\sum_{k=0}^{\infty}(-1)^{k} z^{k}$

The following is an important theorem used in this paper.

\subsection{Differentiation Term by Term Theorem ([23])}

For all non-negative integer $k$, if the functions $g_{k}:(a, b) \rightarrow R$ satisfy the following three conditions : (i) there exists a point $x_{0} \in(a, b)$ such that $\sum_{k=0}^{\infty} g_{k}\left(x_{0}\right)$ is convergent, (ii) all functions $g_{k}(x)$ are differentiable on open interval $(a, b)$, (iii) $\sum_{k=0}^{\infty} \frac{d}{d x} g_{k}(x)$ is uniformly convergent on $(a, b)$. Then $\sum_{k=0}^{\infty} g_{k}(x)$ is uniformly convergent and differentiable on $(a, b)$. Moreover, its derivative $\frac{d}{d x} \sum_{k=0}^{\infty} g_{k}(x)=\sum_{k=0}^{\infty} \frac{d}{d x} g_{k}(x)$.

Before deriving our major results, we need a lemma.

\subsection{Lemma}

Suppose $z$ is a complex number, $n$ is any positive integer, $m$ is any integer, $a$ is a real number, $a \neq 0$ and $|z| \neq|a|^{1 / n}$. Then

$$
\begin{gathered}
\frac{z^{m}}{a+z^{n}} \\
=\sum_{k=0}^{\infty} \frac{(-1)^{k}}{a^{k+1}} z^{n k+m} \quad \text { if }|z|<|a|^{1 / n} \\
=\sum_{k=0}^{\infty}(-1)^{k} a^{k} z^{m-n k-n} \quad \text { if }|z|>|a|^{1 / n}
\end{gathered}
$$

\subsubsection{Proof}

If $|z|<|a|^{1 / n}$, then

$$
\begin{gathered}
\frac{z^{m}}{a+z^{n}} \\
=z^{m} \cdot \frac{1}{a} \cdot \frac{1}{1+\frac{z^{n}}{a}} \\
=z^{m} \cdot \frac{1}{a} \cdot \sum_{k=0}^{\infty}(-1)^{k}\left(\frac{z^{n}}{a}\right)^{k}
\end{gathered}
$$

(By geometric series)

$$
=\sum_{k=0}^{\infty} \frac{(-1)^{k}}{a^{k+1}} z^{n k+m}
$$

If $|z|>|a|^{1 / n}$, then

$$
\begin{gathered}
\frac{z^{m}}{a+z^{n}} \\
=z^{m} \cdot \frac{1}{z^{n}} \cdot \frac{1}{1+\frac{a}{z^{n}}} \\
=z^{m-n} \cdot \sum_{k=0}^{\infty}(-1)^{k}\left(\frac{a}{z^{n}}\right)^{k}
\end{gathered}
$$

(By geometric series)

$$
=\sum_{k=0}^{\infty}(-1)^{k} a^{k} z^{m-n k-n}
$$

q.e.d.

The following is the first result in this study, we determine the Fourier series expansions of any order derivatives of trigonometric function (1).

\subsection{Theorem 1}

Let $a, b, c, \lambda$ be real numbers, $a, \lambda \neq 0, m$ be any integer, $n, p$ be any positive integer, and $|\lambda| \neq|a|^{1 / n}$. Suppose the domain of the trigonometric function

$$
f(x)=\frac{a \cos [m(b x+c)]+\lambda^{n} \cos [(m-n)(b x+c)]}{2 a \lambda^{n} \cos [n(b x+c)]+a^{2}+\lambda^{2 n}}
$$

is $(-\infty, \infty)$.

Case (A): If $|\lambda|<|a|^{1 / n}$, then the $p$-th order derivative of $f(x)$,

$$
f^{(p)}(x)
$$




$$
=b^{p} \cdot \sum_{k=0}^{\infty} \frac{(-1)^{k} \lambda^{n k}(n k+m)^{p}}{a^{k+1}} \cos \left[(n k+m)(b x+c)+\frac{p \pi}{2}\right]
$$

Case (B): If $|\lambda|>|a|^{1 / n}$, then

$$
\begin{gathered}
f^{(p)}(x) \\
=b^{p} \cdot \sum_{k=0}^{\infty} \frac{(-1)^{k} a^{k}(m-n k-n)^{p}}{\lambda^{n k+n}} \cos \left[(m-n k-n)(b x+c)+\frac{p \pi}{2}\right]
\end{gathered}
$$

\subsubsection{Proof}

Let $z=\lambda e^{i(b x+c)}$, then by Euler's formula, we obtain

$$
\begin{gathered}
=\frac{z^{m}}{a+z^{n}} \\
=\frac{\lambda^{m} e^{i m(b x+c)}}{a+\lambda^{n} e^{i n(b x+c)}} \\
=\frac{\lambda^{m} e^{i m(b x+c)}}{\left[a+\lambda^{n} \cos [n(b x+c)]\right]+i \lambda^{n} \sin [n(b x+c)]} \\
=\frac{\lambda^{m} e^{i m(b x+c)}\left\{\left[a+\lambda^{n} \cos [n(b x+c)]\right]-i \lambda^{n} \sin [n(b x+c)]\right\}}{\left.2 a \lambda^{n} \cos [n(b x+c)]\right]^{2}+\lambda^{2 n} \sin ^{2}[n(b x+c)]} \\
\left\{\left[a+\lambda^{n} \cos [n(b x+c)]\right]-i \lambda^{n} \sin [n(b x+c)]+a^{2}+\lambda^{2 n}\right.
\end{gathered}
$$

Case (A): If $|\lambda|<|a|^{1 / n}$, i.e., $|z|<|a|^{1 / n}$. Then

$$
f(x)
$$

$$
=\frac{a \cos [m(b x+c)]+\lambda^{n} \cos [(m-n)(b x+c)]}{2 a \lambda^{n} \cos [n(b x+c)]+a^{2}+\lambda^{2 n}}
$$

$$
=\operatorname{Re}\left(\frac{e^{i m(b x+c)}\left\{\left[a+\lambda^{n} \cos [n(b x+c)]\right]-i \lambda^{n} \sin [n(b x+c)]\right\}}{2 a \lambda^{n} \cos [n(b x+c)]+a^{2}+\lambda^{2 n}}\right)
$$

$$
=\frac{1}{\lambda^{m}} \cdot \operatorname{Re}\left(\frac{z^{m}}{a+z^{n}}\right)
$$

$$
=\frac{1}{\lambda^{m}} \cdot \operatorname{Re}\left[\sum_{k=0}^{\infty} \frac{(-1)^{k}}{a^{k+1}} z^{n k+m}\right]
$$

(By (3) of Lemma)

$$
\begin{array}{r}
=\operatorname{Re}\left[\sum_{k=0}^{\infty} \frac{(-1)^{k} \lambda^{n k}}{a^{k+1}} e^{i[(n k+m)(b x+c)]}\right] \\
=\sum_{k=0}^{\infty} \frac{(-1)^{k} \lambda^{n k}}{a^{k+1}} \cos [(n k+m)(b x+c)]
\end{array}
$$

(By Euler's formula)

Therefore, by differentiation term by term theorem, differentiating $p$-times with respect to $x$ on both sides of (8), we

obtain the $p$-th order derivative of $f(x)$,

$$
f^{(p)}(x)
$$

$$
=b^{p} \cdot \sum_{k=0}^{\infty} \frac{(-1)^{k} \lambda^{n k}(n k+m)^{p}}{a^{k+1}} \cos \left[(n k+m)(b x+c)+\frac{p \pi}{2}\right]
$$

Case (B): If $|\lambda|>|a|^{1 / n}$, i.e., $|z|>|a|^{1 / n}$. The same proof as Case (A), and using (4) of Lemma and (7), we have

$$
f(x)
$$

$$
\begin{gathered}
=\frac{a \cos [m(b x+c)]+\lambda^{n} \cos [(m-n)(b x+c)]}{2 a \lambda^{n} \cos [n(b x+c)]+a^{2}+\lambda^{2 n}} \\
=\operatorname{Re}\left[\sum_{k=0}^{\infty}(-1)^{k} a^{k} \lambda^{-n k-n} e^{i[(m-n k-n)(b x+c)]}\right] \\
=\sum_{k=0}^{\infty} \frac{(-1)^{k} a^{k}}{\lambda^{n k+n}} \cos [(m-n k-n)(b x+c)]
\end{gathered}
$$

Also, by differentiation term by term theorem, differentiating $p$-times with respect to $x$ on both sides of (9), we obtain

$$
\begin{gathered}
f^{(p)}(x) \\
=b^{p} \cdot \sum_{k=0}^{\infty} \frac{(-1)^{k} a^{k}(m-n k-n)^{p}}{\lambda^{n k+n}} \cos \left[(m-n k-n)(b x+c)+\frac{p \pi}{2}\right] \\
\text { q.e.d. }
\end{gathered}
$$

The following is the second major result in this paper, we obtain the Fourier series expansions of any order derivatives of trigonometric function (2).

\subsection{Theorem 2}

Let the assumptions be the same as Theorem 1. Suppose the domain of the trigonometric function

$$
g(x)=\frac{a \sin [m(b x+c)]+\lambda^{n} \sin [(m-n)(b x+c)]}{2 a \lambda^{n} \cos [n(b x+c)]+a^{2}+\lambda^{2 n}}
$$


is $(-\infty, \infty)$.

Case (A): If $|\lambda|<|a|^{1 / n}$, then the $p$-th order derivative of $g(x)$,

$$
\begin{gathered}
g^{(p)}(x) \\
=b^{p} \cdot \sum_{k=0}^{\infty} \frac{(-1)^{k} \lambda^{n k}(n k+m)^{p}}{a^{k+1}} \sin \left[(n k+m)(b x+c)+\frac{p \pi}{2}\right]
\end{gathered}
$$

Case (B): If $|\lambda|>|a|^{1 / n}$, then

$$
\begin{gathered}
g^{(p)}(x) \\
=b^{p} \cdot \sum_{k=0}^{\infty} \frac{(-1)^{k} a^{k}(m-n k-n)^{p}}{\lambda^{n k+n}} \sin \left[(m-n k-n)(b x+c)+\frac{p \pi}{2}\right]
\end{gathered}
$$

\subsubsection{Proof}

Case (A): If $|\lambda|<|a|^{1 / n}$, i.e., $|z|<|a|^{1 / n}$. Using (3) of Lemma, (7), and the same proof as Theorem 1, we have

$$
\begin{gathered}
g(x) \\
=\frac{a \sin [m(b x+c)]+\lambda^{n} \sin [(m-n)(b x+c)]}{2 a \lambda^{n} \cos [n(b x+c)]+a^{2}+\lambda^{2 n}} \\
=\operatorname{Im}\left(\frac{e^{i m(b x+c)}\left\{\left[a+\lambda^{n} \cos [n(b x+c)]\right]-i \lambda^{n} \sin [n(b x+c)]\right\}}{2 a \lambda^{n} \cos [n(b x+c)]+a^{2}+\lambda^{2 n}}\right) \\
=\operatorname{Im}\left[\sum_{k=0}^{\infty} \frac{(-1)^{k} \lambda^{n k}}{a^{k+1}} e^{i[(n k+m)(b x+c)]}\right] \\
=\sum_{k=0}^{\infty} \frac{(-1)^{k} \lambda^{n k}}{a^{k+1}} \sin [(n k+m)(b x+c)]
\end{gathered}
$$

Thus, by differentiation term by term theorem, differentiating $p$-times with respect to $x$ on both sides of (12), we obtain the $p$-th order derivative of $g(x)$,

$$
\begin{gathered}
g^{(p)}(x) \\
=b^{p} \cdot \sum_{k=0}^{\infty} \frac{(-1)^{k} \lambda^{n k}(n k+m)^{p}}{a^{k+1}} \sin \left[(n k+m)(b x+c)+\frac{p \pi}{2}\right]
\end{gathered}
$$

Case (B): If $|\lambda|>|a|^{1 / n}$, i.e., $|z|>|a|^{1 / n}$. By (4) of Lemma and (7), we get $g(x)$

$$
\begin{gathered}
=\frac{a \sin [m(b x+c)]+\lambda^{n} \sin [(m-n)(b x+c)]}{2 a \lambda^{n} \cos [n(b x+c)]+a^{2}+\lambda^{2 n}} \\
\quad=\operatorname{Im}\left[\sum_{k=0}^{\infty}(-1)^{k} a^{k} \lambda^{-n k-n} e^{i[(m-n k-n)(b x+c)]}\right] \\
=\sum_{k=0}^{\infty} \frac{(-1)^{k} a^{k}}{\lambda^{n k+n}} \sin [(m-n k-n)(b x+c)]
\end{gathered}
$$

Hence, by differentiation term by term theorem, differentiating $p$-times with respect to $x$ on both sides of (13), we obtain the $p$-th order derivative of $g(x)$,

$$
\begin{aligned}
& g^{(p)}(x) \\
&= b^{p} \cdot \sum_{k=0}^{\infty} \frac{(-1)^{k} a^{k}(m-n k-n)^{p}}{\lambda^{n k+n}} \sin \left[(m-n k-n)(b x+c)+\frac{p \pi}{2}\right] \\
& \text { q.e.d. }
\end{aligned}
$$

\section{Examples}

In the following, for the differential problem of the two types of trigonometric functions in this study, we provide some examples and use Theorems 1, 2 to determine the Fourier series expansions of any order derivatives and evaluate some higher order derivative values of these trigonometric functions. On the other hand, we employ Maple to calculate the approximations of these higher order derivative values and their solutions for verifying our answers.

\subsection{Example 1}

In Theorem 1, taking $a=3, b=1, c=5, \lambda=1, m=4, n=3$, we obtain the trigonometric function

$$
f_{1}(x)=\frac{3 \cos (4 x+20)+\cos (x+5)}{6 \cos (3 x+15)+10}
$$

Therefore, by Case (A) of Theorem 1, we obtain any $p$-th order derivative of $f_{1}(x)$,

$$
\begin{aligned}
& f_{1}^{(p)}(x) \\
& =\sum_{k=0}^{\infty} \frac{(-1)^{k}(3 k+4)^{p}}{3^{k+1}} \cos \left[(3 k+4)(x+5)+\frac{p \pi}{2}\right] \\
& \text { for all } x \in(-\infty, \infty) .
\end{aligned}
$$

Hence, we can determine the 5 -th order derivative value of $f_{1}(x)$ at $x=-4$, 


$$
f_{1}^{(5)}(-4)=-\sum_{k=0}^{\infty} \frac{(-1)^{k}(3 k+4)^{5}}{3^{k+1}} \sin (3 k+4)
$$

In the following, we use Maple to verify the correctness of (16).

$>\mathrm{f} 1:=\mathrm{x}->(3 * \cos (4 * \mathrm{x}+20)+\cos (\mathrm{x}+5)) /(6 * \cos (3 * \mathrm{x}+15)+10)$;

$$
f 1:=x \rightarrow \frac{3 \cos (4 x+20)+\cos (x+5)}{6 \cos (3 x+15)+10}
$$

>evalf((D@@(f1)(-4),20);

\subsection{0}

$>$ evalf $\left(-\operatorname{sum}\left((-1)^{\wedge} \mathrm{k}^{*}\left(3^{*} \mathrm{k}+4\right)^{\wedge} 5 / 3^{\wedge}(\mathrm{k}+1) * \sin (3 * \mathrm{k}+4), \mathrm{k}=0\right.\right.$. in finity),24);

6251.14412550879173924935

\subsection{Example 2}

In Theorem 1, taking $a=1, b=4, c=2, \lambda=2, m=3, n=1$, we obtain the trigonometric function

$$
f_{2}(x)=\frac{\cos (12 x+6)+2 \cos (8 x+4)}{4 \cos (4 x+2)+5}
$$

Thus, by Case (B) of Theorem 1, we have any $p$-th order derivative of $f_{2}(x)$,

$$
\begin{aligned}
& f_{2}^{(p)}(x) \\
= & 4^{p} \cdot \sum_{k=0}^{\infty} \frac{(-1)^{k}(2-k)^{p}}{2^{k+1}} \cos \left[(2-k)(4 x+2)+\frac{p \pi}{2}\right]
\end{aligned}
$$

for all $x \in(-\infty, \infty)$.

Hence, we can determine the 6-th order derivative value of $f_{2}(x)$ at $x=-\frac{3}{4}$,

$$
\left.f_{2}{ }^{6}\right)\left(-\frac{3}{4}\right)=-4^{6} \cdot \sum_{k=0}^{\infty} \frac{(-1)^{k}(2-k)^{6}}{2^{k+1}} \cos (k-2)
$$

Next, we use Maple to verify the correctness of (19).

$>\mathrm{f} 2:=\mathrm{x}->(\cos (12 * \mathrm{x}+6)+2 * \cos (8 * \mathrm{x}+4)) /(4 * \cos (4 * \mathrm{x}+2)+5)$;

$$
f 2:=x \rightarrow \frac{\cos (12 x+6)+2 \cos (8 x+4)}{4 \cos (4 x+2)+5}
$$

>evalf((D@@6)(f2)(-3/4),16);

\subsection{0}

$>\operatorname{evalf}\left(-4^{\wedge} 6^{*} \operatorname{sum}\left((-1)^{\wedge} \mathrm{k}^{*}(2-\mathrm{k})^{\wedge} 6 / 2^{\wedge}(\mathrm{k}+1)^{*} \cos (\mathrm{k}-2), \mathrm{k}=0\right.\right.$..infi nity),16);

\subsection{Example 3}

In Theorem 2, let $a=4, b=2, c=3, \lambda=1, m=-1, n=4$, we get the following trigonometric function

$$
g_{1}(x)=\frac{4 \sin (-2 x-3)+\sin (-10 x-15)}{8 \cos (8 x+12)+17}
$$

Hence, by Case (A) of Theorem 2, we obtain any $p$-th order derivative of $g_{1}(x)$,

$$
\begin{gathered}
g_{1}{ }^{(p)}(x) \\
=2^{p} \cdot \sum_{k=0}^{\infty} \frac{(-1)^{k}(4 k-1)^{p}}{4^{k+1}} \sin \left[(4 k-1)(2 x+3)+\frac{p \pi}{2}\right]
\end{gathered}
$$

for all $x \in(-\infty, \infty)$.

So we can determine the 8 -th order derivative value of $g_{1}(x)$ at $x=-\frac{5}{4}$,

$$
g_{1}{ }^{(8)}\left(-\frac{5}{4}\right)=2^{8} \cdot \sum_{k=0}^{\infty} \frac{(-1)^{k}(4 k-1)^{8}}{4^{k+1}} \sin \left(2 k-\frac{1}{2}\right)
$$

Using Maple to verify the correctness of (22) as follows: $>\mathrm{g} 1:=\mathrm{x}->(4 * \sin (-2 * \mathrm{x}-3)+\sin (-10 * \mathrm{x}-15)) /\left(8^{*} \cos (8 * \mathrm{x}+12)+1\right.$ 7);

$$
g 1:=x \rightarrow \frac{4 \sin (-2 x-3)+\sin (-10 x-15)}{8 \cos (8 x+12)+17}
$$

>evalf((D@@)(g1)(-5/4),18);

$$
-3.97620345974859071 \cdot 10^{8}
$$

$>\operatorname{evalf}\left(2^{\wedge} 8^{*} \operatorname{sum}\left((-1)^{\wedge} \mathrm{k}^{*}\left(4^{*} \mathrm{k}-1\right)^{\wedge} 8 / 4^{\wedge}(\mathrm{k}+1) * \sin (2 * \mathrm{k}-1 / 2), \mathrm{k}=\right.\right.$ 0. infinity),18);

$$
-3.97620345974859031 \cdot 10^{8}
$$

\subsection{Example 4}

In Theorem 2, let $a=1, b=3, c=1, \lambda=3, m=-2, n=2$, we obtain the trigonometric function

$$
g_{2}(x)=\frac{\sin (-6 x-2)+9 \sin (-12 x-4)}{18 \cos (6 x+2)+82}
$$

Thus, by Case (B) of Theorem 2, we can determine any $p$ -th order derivative of $g_{2}(x)$,

$$
\begin{gathered}
g_{2}{ }^{(p)}(x) \\
=3^{p} \cdot \sum_{k=0}^{\infty} \frac{(-1)^{k}(-4-2 k)^{p}}{3^{2 k+2}} \sin \left[(-4-2 k)(3 x+1)+\frac{p \pi}{2}\right]
\end{gathered}
$$

for all $x \in(-\infty, \infty)$.

Hence, we obtain the 5-th order derivative value of 


$$
\begin{aligned}
& g_{2}(x) \text { at } x=-\frac{1}{2}, \\
& g_{2}{ }^{(5)}\left(-\frac{1}{2}\right)=3^{5} \cdot \sum_{k=0}^{\infty} \frac{(-1)^{k}(-4-2 k)^{5}}{3^{2 k+2}} \cos (k+2)
\end{aligned}
$$

We also employ Maple to verify the correctness of (25). $>\mathrm{g} 2:=\mathrm{x}->(\sin (-6 * \mathrm{x}-2)+9 * \sin (-12 * \mathrm{x}-4)) /(18 * \cos (6 * \mathrm{x}+2)+82)$ ;

$$
g 2:=x \rightarrow \frac{\sin (-6 x-2)+9 \sin (-12 x-4)}{18 \cos (6 x+2)+82}
$$

>evalf((D@@5)(g2)(-1/2),16);

$$
-4196.92583152140
$$

$>\operatorname{evalf}\left(3^{\wedge} 5^{*} \operatorname{sum}\left((-1)^{\wedge} \mathrm{k}^{*}\left(-4-2^{*} \mathrm{k}\right)^{\wedge} 5 / 3^{\wedge}(2 * \mathrm{k}+2) * \cos (\mathrm{k}+2), \mathrm{k}=\right.\right.$ 0. infinity), 16);

\section{$-4196.925831521406$}

\section{Conclusion}

As mentioned, the differentiation term by term theorem plays a significant role in the theoretical inferences of this study. In fact, the application of this theorem is extensive, and can be used to easily solve many difficult problems; we endeavor to conduct further studies on related applications. On the other hand, Maple also plays a vital assistive role in problem-solving. In the future, we will extend the research topic to other calculus and engineering mathematics problems and solve these problems by using Maple. These results will be used as teaching materials for Maple on education and research to enhance the connotations of calculus and engineering mathematics.

\section{REFERENCES}

[1] D. Richards, Advanced Mathematical Methods with Maple, New York: Cambridge University Press, 2002.

[2] C. Tocci and S. G. Adams, Applied Maple for Engineers and Scientists, Boston: Artech House, 1996.

[3] F. Garvan, The Maple Book, London: Chapman \& Hall/CRC, 2001.

[4] M. L. Abell and J. P. Braselton, Maple by Example, 3rd ed., New York: Elsevier Academic Press, 2005.

[5] R. J. Stroeker and J. F. Kaashoek, Discovering Mathematics with Maple : An Interactive Exploration for Mathematicians, Engineers and Econometricians, Basel: Birkhauser Verlag, 1999.

[6] J. S. Robertson, Engineering Mathematics with Maple, New York: McGraw-Hill, 1996.
[7] C. T. J. Dodson and E. A. Gonzalez, Experiments in Mathematics Using Maple, New York: Springer-Verlag, 1995.

[8] S. Araci, D. Gao, and M. Acikgoz, The Frobenius-Euler function and its applications, Korean Annals of Mathematics, Vol. 29, No. 1, pp. 47-55, 2012.

[9] M. A. Patterson, M. Weinstein, andA. V. Rao, An efficient overloaded method for computing derivatives of mathematical functions in MATLAB, ACM Transactions on Mathematical Software (TOMS), Vol. 39, Issue. 3, 2013.

[10] M. Wagner, A. Walther, and B. J. Schaefer, On the efficient computation of high-order derivatives for implicitly defined functions, Computer Physics Communications, Vol. 181, Issue. 4, pp. 756-764, 2010.

[11] A. Griewank and A. Walther, Evaluating Derivatives: Principlesand Techniques of Algorithmic Differentiation, 2nd ed., SIAM, Philadelphia, 2008.

[12] C. - H. Yu, The differential problem of some functions, International Journal of Computer Science and Mobile Applications, Vol. 1, Issue. 1, pp. 31-38, 2013.

[13] C. -H. Yu, A study on the differential problems using Maple, International Journal of Computer Science and Mobile Computing, Vol. 2, Issue. 7, pp. 7-12, 2013.

[14] C. -H. Yu, Using Maple to evaluate the derivatives of some functions, International Journal of Research in Computer Applications and Robotics, Vol. 1, Issue. 4, pp. 23-31, 2013.

[15] C. -H. Yu, Evaluating the derivatives of two types of functions, International Journal of Computer Science and Mobile Computing, Vol. 2, Issue. 7, pp. 108-113, 2013.

[16] C. - H. Yu, Evaluating the derivatives of trigonometric functions with Maple, International Journal of Research in Computer Applications and Robotics, Vol. 1, Issue. 4, pp. 23-28, 2013.

[17] C. - H. Yu, The differential problem of two types of functions, International Journal of Computer Science and Mobile Computing, Vol. 2, Issue. 7, pp. 137-145, 2013.

[18] C. -H. Yu, A Study on the differential problem, International Journal of Research in Aeronautical and Mechanical Engineering, Vol. 1, Issue. 3, pp. 52-57, 2013.

[19] C. -H. Yu, The derivatives of some functions, International Journal of Research in Information Technology, Vol. 1, Issue. 8, pp. 15-23, 2013.

[20] C. -H. Yu, A study on some differential problems with Maple, Proceedings of the 6th IEEE/International Conference on Advanced Infocomm Technology, No. 00291, 2013.

[21] C. -H. Yu, Application of Maple on solving some differential problems, Proceedings of IIE Asian Conference 2013, Vol. 1, pp. 585-592, 2013.

[22] C. - H. Yu, The differential problem of four types of functions, Journal of Kang-Ning, vol. 15, pp. 51-63, 2013.

T. M. Apostol, Mathematical Analysis, 2nd ed., Boston: Addison-Wesley, p230, 1975. 